\title{
Integrative analysis of loss-of-function variants in clinical and genomic data reveals novel genes associated with cardiovascular traits
}

Benjamin S. Glicksberg 1,2,14, Letizia Amadori ${ }^{1,3}$, Nicholas K. Akers ${ }^{1}$, Katyayani Sukhavasi ${ }^{4}$, Oscar Franzén ${ }^{1,5,15}$, Li Li ${ }^{1,2}$, Gillian M. Belbin ${ }^{1,6}$, Kristin L. Akers ${ }^{1,7}$, Khader Shameer ${ }^{1,2}$, Marcus A. Badgeley ${ }^{1,2}$, Kipp W. Johnson ${ }^{1,2}$, Ben Readhead ${ }^{1,2}$, Bruce J. Darrow ${ }^{3}$, Eimear E. Kenny ${ }^{6,8}$, Christer Betsholtz $^{9}$, Raili Ermel ${ }^{10}$, Josefin Skogsberg ${ }^{11}$, Arno Ruusalepp ${ }^{5,9}$, Eric E. Schadt ${ }^{1,2,5,7}$, Joel T. Dudley ${ }^{1,2,12}$, Hongxia Ren ${ }^{13}$, Jason C. Kovacic ${ }^{3}$, Chiara Giannarelli, ${ }^{1,3}$, Shuyu D. Li ${ }^{1,7^{*}}$, Johan L. M. Björkegren ${ }^{1,4,5,11^{*}}$ and Rong Chen ${ }^{1,7^{*}}$

From Varl-COSI 2018: identification and annotation of genetic variants in the context of structure, function, and disease Chicago, IL, USA. 08 July 2018

\begin{abstract}
Background: Genetic loss-of-function variants (LoFs) associated with disease traits are increasingly recognized as critical evidence for the selection of therapeutic targets. We integrated the analysis of genetic and clinical data from 10,511 individuals in the Mount Sinai BioMe Biobank to identify genes with loss-of-function variants (LoFs) significantly associated with cardiovascular disease (CVD) traits, and used RNA-sequence data of seven metabolic and vascular tissues isolated from 600 CVD patients in the Stockholm-Tartu Atherosclerosis Reverse Network Engineering Task (STARNET) study for validation. We also carried out in vitro functional studies of several candidate genes, and in vivo studies of one gene.

Results: We identified LoFs in 433 genes significantly associated with at least one of 10 major CVD traits. Next, we used RNA-sequence data from the STARNET study to validate 115 of the 433 LoF harboring-genes in that their expression levels were concordantly associated with corresponding CVD traits. Together with the documented hepatic lipid-lowering gene, APOC3, the expression levels of six additional liver LoF-genes were positively associated with levels of plasma lipids in STARNET. Candidate LoF-genes were subjected to gene silencing in HepG2 cells with marked overall effects on cellular LDLR, levels of triglycerides and on secreted APOB100 and PCSK9. In addition, we identified novel LoFs in DGAT2 associated with lower plasma cholesterol and glucose levels in BioMe that were also confirmed in STARNET, and showed a selective DGAT2-inhibitor in C57BL/6 mice not only significantly lowered fasting glucose levels but also affected body weight.

(Continued on next page)
\end{abstract}

\footnotetext{
* Correspondence: shuyudan.li@mssm.edu; johan.bjorkegren@mssm.edu; rong.chen@mssm.edu

${ }^{1}$ Department of Genetics and Genomic Sciences, The Icahn Institute for Genomics and Multiscale Biology, Icahn School of Medicine at Mount Sinai, One Gustave L. Levy Place, New York, NY 10029, USA

Full list of author information is available at the end of the article
}

(c) The Author(s). 2019 Open Access This article is distributed under the terms of the Creative Commons Attribution 4.0 International License (http://creativecommons.org/licenses/by/4.0/), which permits unrestricted use, distribution, and reproduction in any medium, provided you give appropriate credit to the original author(s) and the source, provide a link to the Creative Commons license, and indicate if changes were made. The Creative Commons Public Domain Dedication waiver (http://creativecommons.org/publicdomain/zero/1.0/) applies to the data made available in this article, unless otherwise stated. 
(Continued from previous page)

Conclusion: In sum, by integrating genetic and electronic medical record data, and leveraging one of the world's largest human RNA-sequence datasets (STARNET), we identified known and novel CVD-trait related genes that may serve as targets for CVD therapeutics and as such merit further investigation.

Keywords: Loss-of-function variant, Cardiovascular traits, Genetic association, Integrative data analysis, Target identification and validation, Electronic Medical Records

\section{Background}

The delineation and association of loss-of-function variants (LoFs) with human diseases and phenotypic traits continues to play an increasingly important role in the discovery and validation of novel therapeutic targets. Nonsense mutations in PCSK9 are associated with an $88 \%$ reduction in the risk of coronary heart disease (CHD) [1]. While large clinical trials are ongoing [2], current evidence suggests that PCSK9 inhibitors not only lower LDL cholesterol, but also reduce cardiovascular events [3, 4]. In fact, in the recent published FOURIER trial, the PCSK9 inhibitor Evolocumab used in conjunction with background of statin therapy was shown to significantly reduce the risk of cardiovascular events as well as levels of plasma LDL cholesterol [5]. In another example, LoF mutations in NPC1L1, encoding a transporter involved in the absorption of dietary cholesterol, are associated with reduced incidence of CHD [6], and a small-molecule inhibitor of NPC1L1, ezetimibe, was found to both lower plasma LDL levels and reduce the risk of CHD events [7]. HMGCR, LDLR, and APOC3 are additional examples of genes with LoFs or other genetic variants where carriers show lower levels of plasma LDL or triglycerides and a reduced incidence of CHD [8, 9]. It is also well known that statin therapy targeting HMGCR reduces risk of both primary and recurrent CHD events [10]. Hence, the evidence that human genetics may improve therapeutic target selection is mounting and increasingly recognized. In fact, retrospective analysis shows that for novel targets with human genetic validation, the rate of success in clinical development is increased twofold [11].

Besides the targeted analysis of human LoFs in candidate genes, several systematic surveys of LoFs [12, 13] and their associations with clinical phenotypes [14-18] have been performed including the recent DiscovEHR study [19] where the distribution and clinical impact of LoFs in 50,726 whole exomes were investigated. A common theme for these studies is that LoF-phenotype associations were found both in established disease-trait genes, such as PCSK9 (with plasma LDL levels) and APOC3 (with plasma triglycerides), as well as in novel genes associated with unexpected clinical traits [15, 19]. Thus, systematically discovering LoF-harboring genes associated with clinical traits appears to be an effective approach towards precision medicine [citation: https:// academic.oup.com/hmg/article/27/R1/R56/4969371] by identifying novel disease candidate genes that may prove useful as drug targets.

In the current study, we identified LoF variants with possible implications for cardiovascular disease (CVD) using Mount Sinai BioMe Biobank, established in 2007 in New York City, an ongoing, broadly-consented Electronic Medical Record (EMR)-linked data repository that enrolls patients non-selectively from the Mount Sinai Medical Center. So far, over 34,000 ancestrally diverse participants have been enrolled, of which a subset of 10 , 511 with genotype data were used here. Subjects have been extensively characterized with longitudinal clinical information in EMRs, including disease diagnoses, laboratory test results, and medication history [20, 21]. We have demonstrated successful utility of these data for disease subtyping [22], automated phenotyping [citation: https://www.worldscientific.com/doi/abs/10.1142/ 9789813235533_0014], comorbidity analyses [23, 24], health assessment via real-time visualization [25], and drug repurposing [26]. To validate CVD-trait gene associations detected in BioMe, we used RNA sequencing (RNAseq) data from blood and six vascular and metabolic tissues in 600 well-characterized coronary artery disease (CAD) patients of the Stockholm-Tartu Atherosclerosis Reverse Network Engineering Task (STARNET) study [27]. A schematic flow of how we identified LoFs in the BioMe Biobank, further evaluated corresponding LoF-genes in STARNET and then undertook in vitro and in vivo functional validation is shown in Fig. 1.

\section{Methods}

\section{BioMe research cohort}

In the current study, we utilized a genotyped biobank cohort within the Icahn School of Medicine at Mount Sinai. The Charles Bronfman Institute for Personalized Medicine (http://icahn.mssm.edu/research/institutes/ ipm) BioMe Biobank is a repository that consists of over 34,000 enrolled participants. Currently, 11,212 individuals are genotyped using the Illumina Human Omni Express Exome Bead-8 BeadChip v1.1, which has 866,864 markers per sample. BioMe actively recruits individuals 


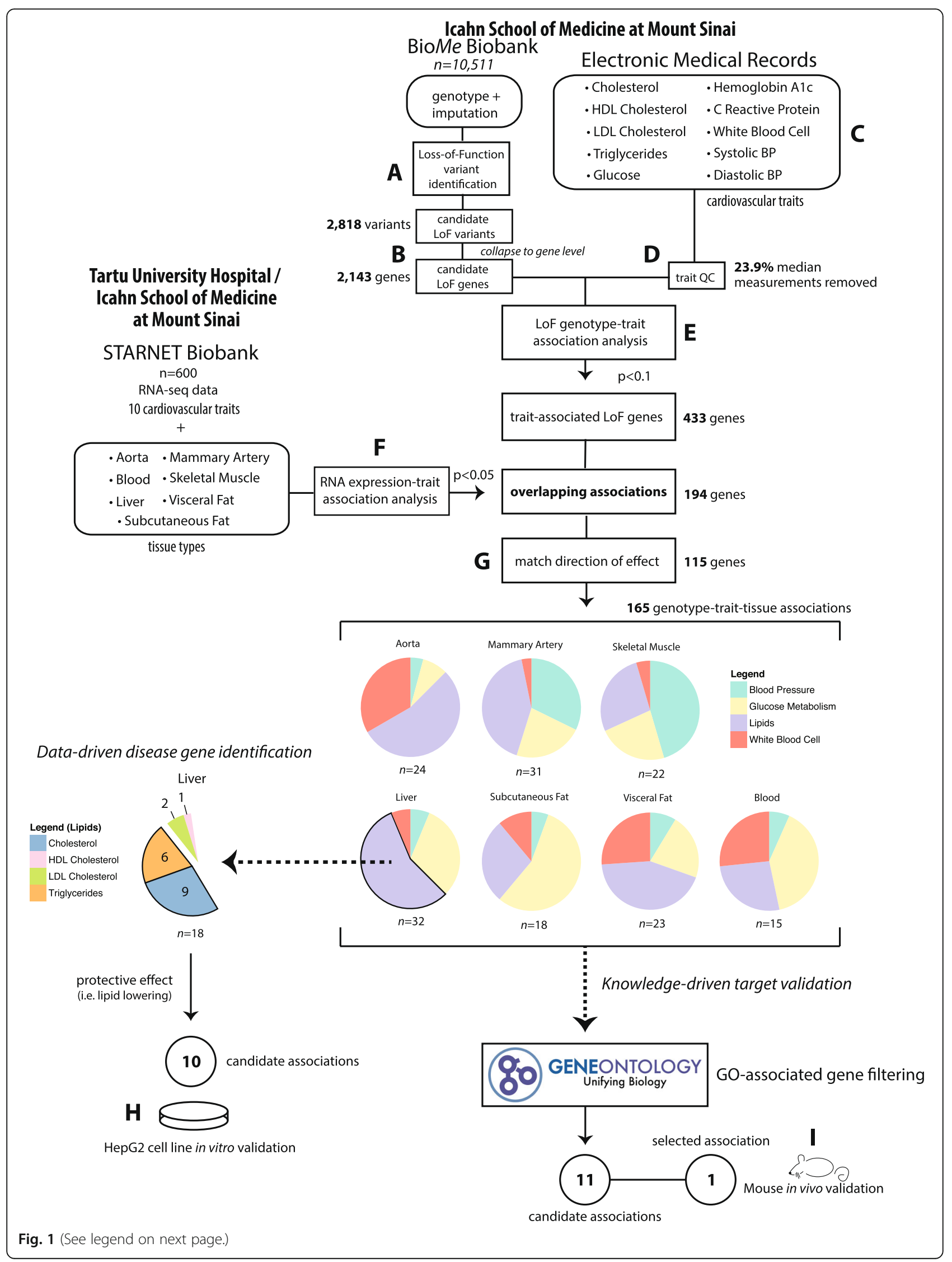


(See figure on previous page.)

Fig. 1 Overall workflow of the study. We first identified high-confidence LoFs in genotyping and the imputed data from the BioMe Biobank (a). Then for each gene with a LoF, we partitioned the BioMe individuals into LoF carriers and non-carriers (b) for comparison of 10 CVD-related traits obtained from the Mount Sinai Hospital Electronic Medical Records (MSH-EMR) (c). Next, we performed trait-specific quality-control (QC) by considering that the CVD-traits are affected by certain ongoing medications (d), followed by statistical analyses to robustly identify LoF-genes that were significantly associated with at least one of these CVD traits (e). In the next step (f), we assessed LoF-harboring genes associated with any of the CVD traits by exploring associations between RNA expression levels of these genes and corresponding CVD traits across seven tissues in STARNET [27]. We then selected genes with concordant CVD trait-associations in both BioMe and STARNET (i.e., when LoFs in a gene are associated with low values of a CVD trait, low expression of the same gene is also associated with low values of the trait) (g). For LoF genes associated with lower plasma cholesterol or triglyceride levels in BioMe Biobank and STARNET liver data, we carried out functional in vitro evaluation using HepG2 cells (H). Last, a knowledge-driven filtration approach was used for leveraging information in Gene Ontology (GO) to select potential therapeutic targets for validation in mice (i)

from upper Manhattan facilitating strong representation of ethnic minorities often underrepresented in other studies. Out of the genotyped individuals, there were 6857 (61.2\%) women and $4350(38.8 \%)$ men, with three individuals removed due to unknown sex. The selfreported ancestry breakdown is as follows: 2090 (18.6\%) European, 3763 African American (33.6\%), 5194 Hispanic/Latino (46.3\%), and 134 Other $(0.01 \%)$. Due to low representation, we merged the remaining ancestry groups into the "Other" category. The mean age for the BioMe participants was $55.8 \pm 15.8$ years.

\section{Genotype imputation, quality control, and filtration}

For the genotype data, all individuals had call rates above 99\% and all SNPs had call rates >95\%, at least one minor allele, and Hardy-Weinberg equilibrium $p$-value $>5 \mathrm{e}-05$. We performed phasing on the genotype data using SHAPEIT v2 (r644) [28] and imputation using IMPUTE2 v2.3 [29] (chunk size of $5 \mathrm{Mb}$ ). For the imputation reference panel, we used the 1000 Genomes [30] Phase 1 version 3 variant set (August, 2012; https:// mathgen.stats.ox.ac.uk/impute/data_download_1000G_

phase1_integrated.html). We then performed several quality control steps on the over 37 million imputed SNP data: 1 . We removed any sites with $<0.3$ gen INFO score (from IMPUTE2); 2. We discarded any variants with $<90 \%$ site completeness; 3 . We removed individual allele call below $95 \%$ genotype probability.

We used PLINK [31] to identify related individuals (relatives) in the cohort that could bias the results of subsequent analyses. We identified 612 pairs of individuals (1224 total) that had PI-HAT scores $>0.2$, which indicates direct relatedness. Accordingly, we randomly selected an individual from each pair to remove from our cohort. After all of the QC steps detailed, the cohort contained 10,511 individuals.

\section{Variant annotation procedure}

As different annotators have their strengths and weaknesses, we decided to utilize multiple annotators to identify predicted LoF mutations. All annotators were run using the hg19 build. Variant Annotation Tool (VAT) is an annotator that was used in the seminal paper by MacArthur et al. [12] to identify LoF mutations. We obtained the VAT annotated file from 1000 genomes [30] $\mathrm{ftp}$ server (ftp://ftp-trace.ncbi.nih.gov/1000genomes/ftp/ phase1/analysis_results/functional_annotation/annotated_vcfs/), specifically phase 1 , version 3 , where full documentation can be found. We limited the variants to those that putatively cause LoF by restricting "effect type" to "frameshift_indel", "stop_gained", or "splice_ site". We also restricted these variants to those with predicted "full" effect. These filtering steps resulted in 4757 VAT-predicted LoF mutations for our dataset.

ANNOVAR is another variant annotator tool utilized in a related follow-up study by Li et al. [15]. Like with the previous annotator, we ran our dataset mimicking the same parameters used in their study to identify LoFs using ANNOVAR (v. 2015Apr14). Specifically, we filtered the resulting "Gene-based Annotation" results for variants causing "frameshift substitution", "stopgain", or "exonic;splicing". We obtained 10,582 predicted LoFs from these filtering steps.

SnpEff [32] is a popular annotation tool that has been used in many studies. To identify LoF mutations, we ran our dataset using this tool (SnpEff v. 3.6) and filtered for SNPs with "HIGH" Effect_Impact, "protein-coding" Transcript_BioType, and the following Effect types: "STOP_ GAINED”, "SPLICE_SITE_ACCEPTOR”, "SPLICE_SITE_ DONOR”, or "FRAME_SHIFT”. We identified 6387 putative LoFs from our dataset using these criteria.

\section{Consensus LoF variant classification}

We only included into our analyses consensus mutations, or mutations in which at least two of three annotators identify as LoF. Additional file 1: Figure S1 demonstrates the relative distribution of LoF annotation between the three annotators. It is important to note that the overall numbers of variants called per annotator might differ from the effect calls per annotator as a particular variant can have more than one effect call. In total, we identified 6421 putative consensus LoF variants. We next performed extensive, further QC curation to ensure high confidence and 
quality LoF annotation. From the imputation-specific QC, we only kept variants that had $>0.3$ gen INFO scores (IMPUTE2; 1666 failed) and $>90 \%$ site completeness using 95\% as call threshold (301 failed). Furthermore, we excluded low confidence alleles with quality scores $<95 \%$. Additionally, we only included variants that: 1) had consensus LoF annotation on at least one overlapping transcript (i.e., only include variant if two or more annotators classified the same associated transcript as LoF; 28 failed); 2) were not in the final exon of the predicted LoF transcript (1498 failed); 3) had $<2 \%$ alternate allele frequency (362 failed). Taken together, these filtering steps resulted in the removal of 2029 unique variants (as a single variant could fail overlapping filtering steps), leaving 3392 unique remaining variants from the original list of 6421. Lastly, we filtered variants in which there were no carriers in our cohort, which produced 2818 high

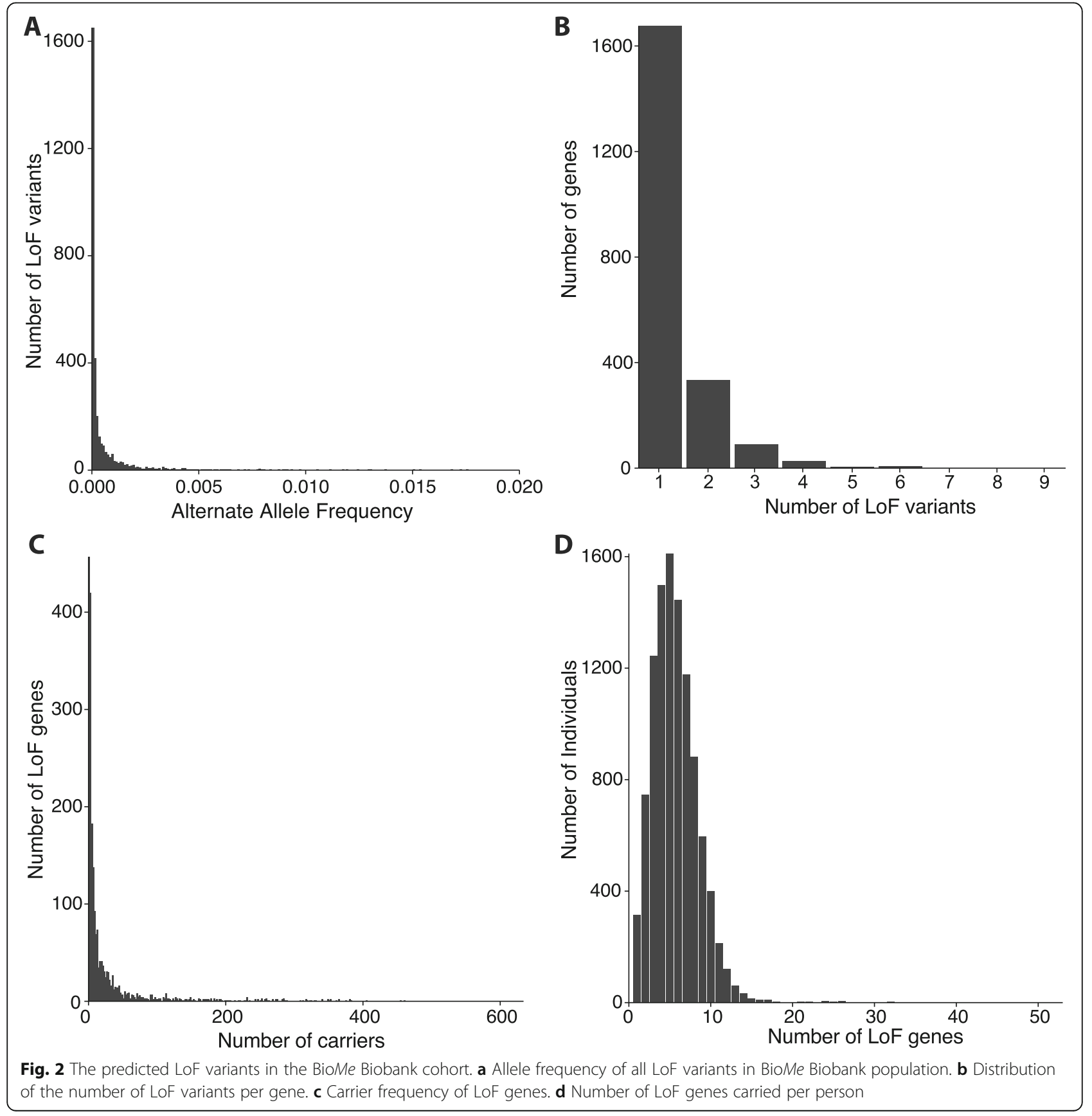


confidence predicted LoF variants for use in our study (see Additional file 1: Figure S2A for variant filtering procedure overview).

As a matter of practicality, we collapsed mutations to the gene level, as the predicted outcome of each independent LoF mutation within a single gene is theoretically identical. The relative distributions of mutations per gene can be seen in Fig. 2b. On average, we found $1.32 \pm 0.73$ (STD) LoF mutations per gene, with a maximum of 9 mutations in the gene PZP, which has 65,552 base pairs. The frequency of these mutations in the population can be seen in Fig. 2c. Specifically, the 2818 variants were collapsed into a useable list of 2143 genes. The distribution of LoF gene frequency in this population can be seen in Fig. 2d. We present all variants used in this study along with their frequency in our cohort and their respective effect call for each annotator in Additional file 2: Table S1.

\section{Trait data collection and processing}

The BioMe Biobank cohort used in this study is connected to the MSH's EMR via de-identified codes managed by the Mount Sinai Data Warehouse. These records contain information on trait measurements and medication prescriptions, which we collected for all of the individuals in the cohort. We selected 10 CVDrelated traits for analysis, specifically: glucose levels, hemoglobin A1c, total cholesterol, LDL cholesterol, HDL cholesterol, triglycerides, high sensitivity $C$ reactive protein, diastolic blood pressure, systolic blood pressure, and white blood cell count. We obtained trait measurements through querying the EMR, which consisted of individual, value, date, and unit of measurement. This cohort, however, was not specifically screened for enrollment criteria. As such, there are many factors that could have extreme effects on the trait values that are irrespective of genetics. Of crucial concern are changes in trait levels due to diseases and/or medication use. While several algorithms exist to control the effect of medications in trait analytics, there is no established, universal methodology. Accordingly we devised a thorough and individualized procedure to control for confounding factors, including disease and medication effects, in the current study, which we describe in detail in the Additional file 1: Supplementary Materials.

In brief, we performed several QC steps on the trait measurement data to ensure the highest achievable validity (Additional file 1: Figure S2B). We filtered out all incorrectly labeled, outlier, and medication/disease-affected measurements, and then combined any remaining measurements into a single median value per person. For each of the 10 traits, we present the exclusion QC process (i.e. number of individuals and total number of measurements before and after filtering) in Additional file 2: Tables S3-S5; specifically due to medication use (Additional file 2: Table S3), outliers (Additional file 2: Table S4), and combined (Additional file 2: Table S5).

\section{Association analysis between LoF genes and traits}

We performed linear regression analysis to test whether having a LoF mutation significantly affects trait measurements. Our analytical goal was to assess if genes harboring LoF genetic variants were significantly associated with CVD traits. Therefore, we chose to perform the analysis at gene level rather than at the level of individual variants. CVD trait values and ages used in the analyses were median values that passed QC steps explained previously. We observed the regression residuals were not approximately normally distributed and thus would not satisfy assumptions of linear regression. Accordingly, we first performed the Box-Cox transformation (Eq. 1) procedure to identify the optimal transformation parameter per trait that produces a normal distribution of residuals by sequencing through $\lambda$ values from the range $[-2,2]$ in increments of 0.5 . The natural logarithm was used for the case of $\lambda=0$. For each trait, we selected the $\lambda$ value corresponding to the highest regression model (Eq. 2 without the gene variable) log-likelihood as the optimal transformation parameter. All regressions were generalized linear models fit to the data using the method of iteratively reweighted least squares. The effects of transformation can be found in Additional file 1: Figure S8.

$$
y_{i}^{(\lambda)}\left\{\begin{array}{cc}
\frac{y_{i}^{(\lambda)}-1}{\lambda} & \text { if } \lambda \neq 0 \\
\ln \left(y_{i}\right) & i f \lambda \neq 0
\end{array}\right.
$$

With the lambda parameter calculated, we then performed the following formula to assess for genotypetrait significance:

$$
\begin{array}{r}
P\left(\text { trait }^{\lambda} \mid \beta_{0}+\beta_{1} \cdot \text { gene }+\beta_{\mathrm{g}} \cdot \text { sex }+\beta_{\mathrm{a}} \cdot\right. \text { age } \\
\left.+\beta_{\mathrm{pc} 1} \cdot \mathrm{PC} 1 \ldots+\ldots \beta_{\mathrm{pc} 5} \cdot \mathrm{PC} 5\right)
\end{array}
$$

where trait is continuous value, $\lambda$ is the transformation parameter factor derived through Box-Cox transformation (Eq. 1), gene is binary piecewise Yes/No indicating presence of LoF mutation, sex is binary piecewise Female/Male, age is continuous constant per year, principal components are continuous.

To obtain an estimate of effect size, for associations that were found to be significant in (Eq. 2), we performed a similar statistical analysis albeit without response transformation and used both $\beta_{1}$ and t-statistic $\left(\beta_{1} / \beta\right.$ standard error). 


\section{RNA-expression data source: STARNET}

The details of the STARNET study have been previously published [27], however in brief: patients diagnosed with CAD undergoing open-heart surgery had up to 7 tissues collected, including aortic root (AOR), whole blood, liver (LIV), mammary artery (MAM), subcutaneous fat (SF), skeletal muscle (SKLM), and visceral fat (VAF). RNA was derived from each tissue and sequenced at read lengths of 50 or 100 base pairs from a single-end. Reads were aligned to human genome hg19 using the STAR aligner [33] version 2.3.0e and GENCODE v19 [34] annotations. Reads aligned to genes were summarized using HTSeq [35]. For each tissue, genes with less than one count per million (CPM) in more than $90 \%$ of samples were filtered. Genes passing this criteria were normalized using voom [36] using the 'TMM' method, generating $\log (\mathrm{CPM})$ values and precision weights. A linear model was then used to adjust for batch effects using sequencing flow cell identifier. An lmFit object was generated with the general formula $\log (\mathrm{CPM}) \sim$ FlowCellID. The residuals of this linear model were used as batch-normalized gene expression values in subsequent analysis.

\section{Association analysis between gene expression and traits}

We compared the RNA expression levels of the identified LoF genes in all STARNET tissues to the same 10 CVD traits. Linear modeling was performed to assess the correlation between gene expression and clinical trait, after adjustment for basic clinical covariates. The model used was: Trait $\sim$ Gene + Age + Gender + BMI.

\section{Integration of BioMe data and STARNET data}

We assumed that LoF variants in BioMe and low gene expression level in STARNET have the same biological and consequently physiological effect. Therefore we defined concordant associations in BioMe and STARNET as follows: when LoFs in a gene are associated with low values of a CVD trait in BioMe, low expression of the same gene is also associated with low values of the trait in STARNET; conversely when LoFs in a gene are associated with high values of a CVD trait in BioMe, low expression of the same gene is also associated with high values of the trait in STARNET. Furthermore, we considered the associations in BioMe and STARNET concordant regardless what tissues in the STARNET data where significant associations between gene expression and CVD-traits were observed. To illustrate the definition of concordant associations in BioMe and STARNET, in Table 2, LoF in gene ACSM3 is associated with low LDL cholesterol level in BioMe, and low gene expression of ACSM3 is also associated with low LDL cholesterol level in skeletal muscle (SKLM) in STARNET; conversely, LoF in gene ACOT11 is associated with high glucose level in BioMe, and low gene expression of ACOT11 is also associated with high glucose level in skeletal muscle (SKLM) in STARNET.

\section{Mouse DGAT2 validation procedure}

In the current study, C57BL/6 wild type male mice (3 months old, fed chow diet) were obtained from the Jackson Laboratory. The animals were maintained in a pathogen-free and temperature-controlled room on a 12 $\mathrm{h}$ light/dark cycle and supplied with food and water ad libitum. Compounds were administered as previously described [37, 38] with modifications, specifically, mice were orally dosed with vehicle (control) or inhibitors 20 $\mathrm{mg} / \mathrm{kg} /$ day. Body weight and fasting glucose were measured 3 days after compound treatment.

\section{siRNA candidate targets validation study}

Cells from the human hepatocellular carcinoma cell line HepG2 were plated in six-well culture dishes (Corning) containing $10 \%$ fetal bovine serum (FBS)-RPMI-1640 medium supplemented with penicillin $(100 \mathrm{U} / \mathrm{mL})$ and streptomycin $(100 \mu \mathrm{g} / \mathrm{mL})$. For each gene, cells were transfected with siRNAs (Ambion, Life Technologies, Additional file 2: Table S9), using Lipofectamine RnaiMax according to the manufacturer's instructions (Invitrogen). Two days after transfection, siRNA-targeted cells and mock-treated controls (scramble siRNA) were incubated with oleic acid ( $0.75 \mathrm{mM}$, Sigma Aldrich,) for $24 \mathrm{~h}$ in $1 \%$ FBS medium. Thereafter, the cells were examined for effects on levels of PCSK9 and LDLR, and on measurements of cholesterol (total cholesterol, TC; and cholesteryl esters, CE) and triglycerides (TG).

Total RNA was isolated from HepG2 cells with the RNeasy Mini-kit (Qiagen) and concentrations determined by NanoDrop (Thermo Scientific). The efficiency of target gene silencing was determined by TaqMan analyses (Additional file 2: Table S9). In brief, cDNA was synthesized from $0.5 \mu \mathrm{g}$ of total RNA (High-Capacity RNA-to-cDNA Kit, Thermo Scientific) and amplified by real-time PCR with 1xTaqMan universal PCR master mix (Applied Biosystems) using primers and probes from Applied Biosystems (Additional file 2: Table S9); data were normalized to mock control using the comparative Ct method.

\section{Cholesterol and triglyceride measurement procedure}

Cholesterol accumulation in cell lysates of siRNA-targeted HepG2 cells was measured using the Total Cholesterol and Cholesteryl Ester Colorimetric/Fluorometric Assay kit (BioVision). Lipid levels were normalized by protein concentrations obtained from the same samples using the BCA method (Thermo Scientific). Triglycerides from siRNA targeted HepG2 cells were determined by an 
enzymatic assay using the Triglyceride Quantification Colorimetric/Fluorometric Kit (BioVision).

\section{ELISA assay to measure PCSK9 and LDLR plasma levels} PCSK9 levels were measured using the Human PCSK9 ELISA kit (Cell Biolabs, INC.), according to manufacturer instructions. LDLR levels in cell lysates were measured using the Human LDLR ELISA kit (R\&D Systems), according to manufacturer instructions. Absorbance for both methods was quantitated on Promega Glomax reader at $450 \mathrm{~nm}$.

\section{Experimental statistical analyses}

All experiments were performed in triplicate from 3 independent experiments; data are shown as mean \pm SEM. Statistical differences were assessed with unpaired 2-tailed Student t-tests. Values of $P<0.05$ were considered statistically significant. Computations were performed with GraphPad Prism 6 software (La Jolla, CA 92037 USA).

\section{Results}

LoF-harboring genes associated with CVD traits in BioMe

Genotyping and imputed data from 11,212 individuals of the BioMe Biobank were used to identify a total of 2818 LoFs in 2143 different genes (Methods; Additional file 1: Figure S1; Additional file 1: Figure S2A). Among the 2818 LoFs, 2117 (75\%) were genotyped in the BioMe data, and the rest of the LoFs have been previously identified through direct sequencing in databases such as 1000 Genomes [30] and ExAC [39] (Additional file 2: Table S1). Most of the identified LoFs (95\%) were rare (allele frequency $<0.5 \%$, Fig. 2a) and most genes (2009/ 2143 [94\%]) had $\leq 2$ LoFs (Fig. 2b). Approximately 60\% of those genes with LoFs had 10 or fewer carriers (Fig. 2c). Among individuals carrying at least one LoF, LoFs were typically found in less than 10 genes (mean 5.7, median 5.0; Fig. 2d).

Next, we selected $10 \mathrm{CVD}$-traits from the MSH-EMR associated with the $\mathrm{BioMe}$ individuals (Fig. 1), namely: glucose levels, hemoglobin A1c, total cholesterol, LDL cholesterol, HDL cholesterol, triglycerides, high sensitivity $\mathrm{C}$ reactive protein, diastolic blood pressure, systolic blood pressure, and white blood cell count. Before analyzing these traits in relation to LoFs, we removed trait data that was associated with ongoing medication use that potentially affected the trait (Supplementary Methods; Supplementary Results; Additional file 1: Figure S2B; Additional file 1: Figure S3-S6; Additional file 2: Table S2-S5). We required there to be at least three carriers of LoFs for a given gene with CVD-trait measurements in order to be analyzed, resulting in 1371 of the original 2143 genes examined for their association with CVD traits (see Methods). A total of 644 significant
LoF-CVD trait associations involving 433 genes were identified (linear regression, $p<0.1$ ) (Fig. 1; Additional file 2: Table S6). $79 \%$ of the LoFs associated with a CVD trait were genotyped. The LoF genes were both associated with higher and lower levels of the CVD traits (Additional file 1: Figure S4). The statistically most significant LoF-CVD trait association was reassuringly found in PCSK9 associated with lower plasma LDL cholesterol $(p=1.1 \mathrm{E}-09, \beta=-43 \mathrm{mg} / \mathrm{dL})$ as well as plasma cholesterol $(p=4.3 \mathrm{E}-07, \beta=-44 \mathrm{mg} / \mathrm{dL}$; Additional file 2: Table S6). We also identified a LoF in APOC3 that was associated with reduced levels of plasma triglycerides $(p=5.6 \mathrm{E}-03, \beta=-60 \mathrm{mg} / \mathrm{dL})$ as expected.

\section{Integrating RNA expression evidence from STARNET to the putative associations}

Limitations of the BioMe Biobank analysis of LoFs revolve mainly around sample size, availability of trait measurements, trait filtration, and the low frequency of most LoFs (see also Discussion). As such, many of the LoF-CVD trait associations may risk being inflated due to a skewed distribution of CVD trait levels in a few LoF carriers. At the same time, sample size issues could mask true associations trending towards significance (hence we used a less stringent cutoff $p<0.1$ ). To address some of these limitations and to seek functional evidence as to what organ the LoF may impact for a given CVD trait, we sought associations between the expression levels of LoF-harboring genes and corresponding CVD traits in RNAseq data from the STARNET study [27] (Fig. 1; see Methods). Of the 644 LoF-CVD trait associations found in BioMe, 241 also had at least one significant $(p<0.05)$ gene expression-CVD trait association in STARNET in at least one of the seven tissues, corresponding to 194 unique genes (Fig. 1). Out of these, 115 unique genes, 134 gene-trait associations and 165 combinations of gene-trait-tissue associations were concordant (Fig. 1; Additional file 2: Table S7), i.e. LoFs associated with lower levels of a CVD trait in BioMe also showed a positive correlation with expression levels in STARNET and vice versa. In the 115 genes with concordant associations, $82 \%$ of the LoFs were genotyped. The discordant significant results between the two data sources, while not considered for follow-up evaluation, could still reflect true biological associations that could not be reliably discerned due to study limitations.

\section{Validation of hepatic gene targets predicted to lower plasma cholesterol and triglyceride levels in an in vitro model}

We selected 10 genes (Table 1) associated with either lower plasma cholesterol or triglyceride levels in both BioMe $(\beta<0, p<0.1)$ and STARNET liver tissue $(\beta>0$, $p<0.05)$ for further experimental validation by 
Table 1 Selection of novel genes involved in regulating cholesterol or triglyceride levels for in vitro functional studies

\begin{tabular}{|c|c|c|c|c|c|c|c|}
\hline Gene & Description & Trait & $\begin{array}{l}p \text {-value } \\
(\text { BioMe) }\end{array}$ & $\begin{array}{l}\beta \\
(\mathrm{BiOMe})\end{array}$ & $\begin{array}{l}p \text {-value } \\
\text { (STARNET) }\end{array}$ & $\begin{array}{l}\beta \\
\text { (STARNET) }\end{array}$ & $\begin{array}{l}\text { Tissue } \\
\text { (STARNET) }\end{array}$ \\
\hline CYP2C19 & Cytochrome P450 Family 2 Subfamily C Member 19 & TC & 0.089 & -32 & 0.033 & 0.10 & LIV \\
\hline$P C K 2$ & $\begin{array}{l}\text { Phosphoenolpyruvate Carboxykinase 2, } \\
\text { Mitochondrial }\end{array}$ & TC & 0.10 & -23 & 0.00053 & 0.55 & LIV \\
\hline RNMTL1 & RNA methyltransferase-like protein 1 & TC & 0.054 & -36 & 0.016 & 0.60 & LIV \\
\hline SCRN2 & Secernin 2 & TC & 0.089 & -26 & 0.0026 & 0.39 & LIV \\
\hline UGT1A4 & UDP Glucuronosyltransferase Family 1A4 & TC & 0.069 & -13 & $2.1 \mathrm{E}-05$ & 0.70 & LIV \\
\hline$A B H D 14 B$ & Abhydrolase Domain Containing 14B & TG & 0.027 & -11 & 0.026 & 0.47 & LIV \\
\hline APOC3 & Apolipoprotein C3 & TG & 0.0056 & -56 & 0.0065 & 0.38 & LIV \\
\hline CES3 & Carboxylesterase 3 & TG & 0.061 & -40 & 0.00011 & 0.42 & LIV \\
\hline NMRAL1 & NmrA-Like Family Domain Containing 1 & TG & 0.080 & -28 & 0.045 & 0.28 & LIV \\
\hline SLC39A5 & Solute Carrier Family 39 Member 5 & TG & 0.092 & -70 & 0.0036 & 0.43 & LIV \\
\hline
\end{tabular}

Negative $\beta$ in BioMe and positive $\beta$ in STARNET indicate LoF and low gene expression are associated with decreased trait measurements LIV liver, TC total cholesterol, TG triglycerides

performing gene silencing using the HepG2 in vitro cellular model of hepatocytes. A hepatocyte model was selected since the liver is the primary organ responsible for regulating systemic cholesterol and triglyceride levels (see below). Five genes, CYP2C19, PCK2, RNMTL1 (MRM3), SCRN2, UGT1A4, associated with lower levels of plasma cholesterol (Fig. 3a) were selected due to their potential role in hepatic cholesterol metabolism for in vitro validation. $P C K 2$ was included at our significance threshold $(p=0.10)$ and UGT1A4 and RNMTL1 were included using lower variant imputation genotype probability scores, making in silico results from these in this analysis more speculative. Validation was performed by measuring intracellular levels of LDLR and in media, PCSK9 and APOB100 after individual silencing by siRNA in HepG2 cells (Fig. 3b). While we observed robust expression levels of PCK2, RNMTL1, and SCRN2 in HepG2 cells, CYP2C19 and UGT1A4 were not expressed at detectable levels and were therefore excluded from the validation. For these experiments, PCK2, RNMTL1 and SCRN2 achieved $>90 \%$ inhibition of gene expression by siRNA (Additional file 2: Table S9). Individual silencing of RNMTL1 and SCRN2 both significantly reduced levels of media APOB100 and PCSK9 and concurrently increased cellular LDLR levels (Fig. 3c). Silencing of PCK2 also reduced media levels of APOB100 and PCSK9 but had no significant effect on LDLR levels (Fig. 3c).

Gene silencing in HepG2 cells was also used to confirm the role of $A P O C 3$, and to assess the function of three novel candidate genes (ABHD14B, NMRAL1, and $S L C 39 A 5)$ found to be associated with lower levels of plasma triglycerides in BioMe and STARNET liver data. CES3 was not selected for further in vitro validation because of its well-documented effect in lowering plasma triglycerides and cholesterol levels in liver-specific $\mathrm{KO}$ mice [40] and in reducing the secretion of ApoB100 and triglyceride by its pharmacological inhibition in human hepatocytes [41]. As the liver has negligible or no role in regulating plasma triglyceride uptake, the roles of the three new hepatic genes $A B H D 14 B$, NMRAL1, and SLC39A5 (if any) most likely are through facilitating hepatic secretion of very-low density lipoproteins (VLDL, Fig. 3a). APOC3 is best known for increasing plasma triglyceride levels by interfering with lipoprotein lipase-mediated hydrolysis of plasma triglyceride-rich VLDL particles on the endothelial surface of peripheral tissues (e.g., in fat). However, recent evidence shows that hepatic APOC3 also facilitates the secretion of VLDL from the liver [42]. Silencing of APOC3 and SLC39A5 in HepG2 cells reduced media levels of APOB100 and triglycerides and at the same time increased intracellular levels of triglycerides, presumably due to secondary accumulation following reduced secretion of VLDL (Fig. 3d). Silencing of NMRAL1 and ABHD14B, respectively, reduced and markedly increased media levels of APOB100 but had no apparent effect on levels of media or cellular triglycerides.

\section{A knowledge-driven approach to identify targets for in vivo validation}

We also applied a knowledge-driven approach to validate potential therapeutic targets in parallel with the data-driven approach (Fig. 1). We filtered the complete list of 115 genes with concordant associations in $\mathrm{BioMe}$ (LoF-CVD trait) and STARNET (expression-CVD trait, in any tissue) using GO annotations. A total of 90 genes $(78.3 \%)$ had at least one GO-associated biological process term. Nine of these 90 genes corresponding to 11 gene-trait associations had at least one GO term related to the CVD traits of interest (e.g., biological processes relating to blood pressure, glucose, cholesterol or 


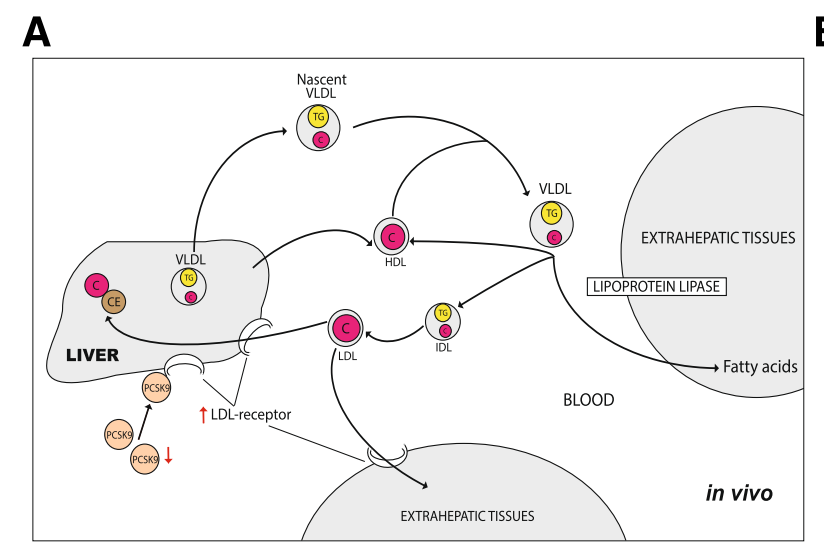

\section{B}

C
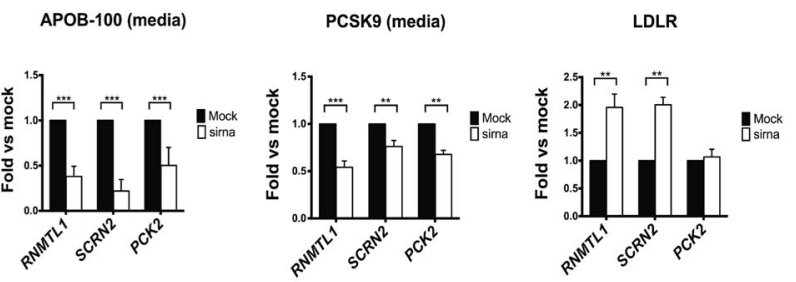

D

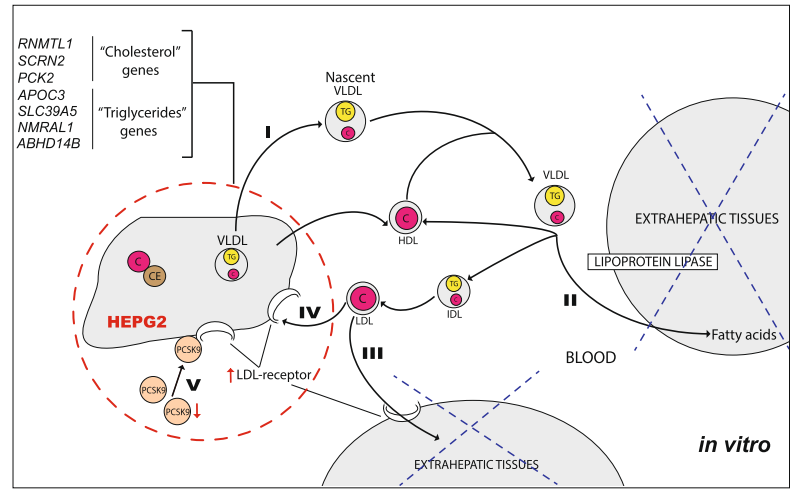

APOB-100 (media)
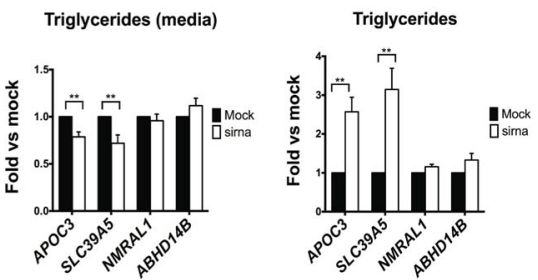

Fig. 3 In vitro validation of candidate genes for lowering plasma cholesterol and triglycerides. a Schematic illustration of lipoprotein metabolism in vivo, and $\mathbf{b}$ An in vitro HepG2 cell model to validate three hepatic plasma cholesterol and three hepatic triglyceride candidate genes. I) Cholesterol- and triglyceride-containing very low density lipoprotein (VLDL) particles are synthesized in, and secreted from, the liver to circulation elevating plasma levels of cholesterol and triglycerides. II) The VLDL particles then travel in blood to microcirculation in peripheral tissues, such as the skeletal muscle and adipose tissue, where lipoprotein lipase $(\mathrm{LPL})$ anchored to the endothelium mediates hydrolysis of VLDL-triglycerides forming free fatty acids that are taken up by the local tissue. This extra-hepatic process lowers plasma triglyceride levels. III) The LPL-mediated hydrolysis of VLDL particles results in the formation of smaller cholesterol-rich low-density lipoproteins (LDL) particles. Some LDL particles are taken up by LDL receptors in extra-hepatic tissues, a process that lowers plasma levels of LDL and cholesterol. IV) The most important regulatory process of plasma LDL and cholesterol levels is, however, the uptake of LDL by hepatic LDL receptors. V) Last, uptake of LDL by the LDL receptor in the liver is inhibited by hepatic synthesis of PCSK9 that binds to the LDL receptors and permits their recirculation away from the hepatocyte cell surface (and LDL receptor degradation) effectively lowering the uptake of LDL particles. Thus, high levels of hepatic PCKS9 lead to reduced LDL uptake and higher plasma cholesterol levels. The HepG2 in vitro model of the liver was chosen as it is the most important organ to control plasma triglyceride and cholesterol levels (a) and since all lipid-associated candidate genes were identified in STARNET liver RNA-seq data. However as illustrated in panels $\mathbf{a}$ and $\mathbf{b}$, an in vitro model of the liver in the form of HepG2 cells cannot fully model lipid metabolism and the extrahepatic tissue contribution in vivo. c For the plasma cholesterol-lowering candidate genes (RNMTL1, SCRN2 and PCK2), Apolipoprotein B-100 (APOB-100), Proprotein Convertase Subtilisin/Kexin type 9 (PCSK9) protein levels were measured in the cell media whereas LDL-receptor (LDLR) was measured in cell lysates. d For the plasma triglyceride-lowering candidate genes (APOC3, SLC39A5, NMRAL1, ABHD14B), Apolipoprotein B-100 (APOB-100), triglycerides (TG) were measured in cell media and lysates. In all experiments, the silencing efficiency of each gene resulted in more than $90 \%$ decrease in gene expression measured after $72 \mathrm{~h}$ incubation, including $24 \mathrm{~h}$ treatment with oleic acid performed before cell harvesting. Values are means \pm SEM. Results are based on 3 biological replicates. $p<0.0332\left(^{*}\right), p<0.0021\left(^{* *}\right), p<0.0002\left(^{* *}\right)$. IDL, intermediate-density lipoprotein. Computational analysis results of the genes tested in (c) and (d) are shown in Additional file 1: Figure $\$ 9$

triglycerides) (Table 2; Additional file 2: Table S8 for GO annotation).

Of these, we selected DGAT2 for follow-up validation for several reasons. First, the DGAT2 expression-CVD trait association for glucose levels was identified in liver (Table 2), a tissue type with more biological relevance to the trait. Second, pharmacological inhibitors of DGAT2 are available (Futatsugi et al. 2015) for further experimental validation. Most importantly, a homolog of $D G A T 2$, DGAT1, has been extensively pursued as a therapeutic target for obesity and diabetes yet with limited clinical success (see below), and we wanted to explore if DGAT2 would be a more viable target.
DGAT2 exhibited significant associations with plasma cholesterol and glucose levels in both BioMe and STARNET (Table 2). In the BioMe Biobank, we identified a stop-gain LoF based on genotyping data (stop codon Y285*, $0.001 \%$ allele frequency) found in 12 heterozygous individuals located in the middle of the enzymatic domain of DGAT2 (Additional file 1: Figure S7A) that likely triggers non-sense mediated decay resulting in no protein synthesis (Additional file 1: Figure S7B and S7C). Individuals with this LoF had significantly $(p<0.1)$ lower plasma glucose (Fig. 4a) and cholesterol (Fig. 4b) levels and statistically non-significant $(p>0.1)$ lower plasma triglycerides 
Table 2 Significant gene-trait associations (BioMe $p<0.1$, STARNET $p<0.05$ ) of genes known to be involved in lipid and glucose metabolism based on GO annotation

\begin{tabular}{|c|c|c|c|c|c|c|c|c|}
\hline & Description & GO BP annotation & Trait & $\begin{array}{l}p \text {-value } \\
\text { (BioMe) }\end{array}$ & $\begin{array}{l}\beta \\
(\mathrm{BioMe})\end{array}$ & $\begin{array}{l}p \text {-value } \\
\text { (STARNET) }\end{array}$ & $\begin{array}{l}\beta \\
\text { (STARNET) }\end{array}$ & $\begin{array}{l}\text { Tissue } \\
\text { (STARNET) }\end{array}$ \\
\hline ACOT11 & Acyl-CoA Thioesterase 11 & $\begin{array}{l}\text { GO:0006631 (fatty acid } \\
\text { metabolic process) }\end{array}$ & Glucose & 0.059 & 40 & 0.040 & -0.28 & SKLM \\
\hline ACSM3 & $\begin{array}{l}\text { Acyl-CoA Synthetase Medium- } \\
\text { Chain Family Member } 3\end{array}$ & $\begin{array}{l}\text { GO:0006631 (fatty acid } \\
\text { metabolic process) }\end{array}$ & $\begin{array}{l}\mathrm{LDL} \\
\text { Cholesterol }\end{array}$ & 0.060 & -4.3 & 0.032 & 0.10 & SKLM \\
\hline$A D C Y 4$ & Adenylate Cyclase 4 & $\begin{array}{l}\text { GO:007137 (cellular response to } \\
\text { glucagon stimulus) }\end{array}$ & Glucose & 0.0095 & 39 & 0.025 & -0.40 & MAM \\
\hline$A D C Y 4$ & Adenylate Cyclase 4 & $\begin{array}{l}\text { GO:007137 (cellular response to } \\
\text { glucagon stimulus) }\end{array}$ & $\begin{array}{l}\text { Hemoglobin } \\
\text { A1C }\end{array}$ & 0.034 & 1.4 & 0.034 & -0.14 & SF \\
\hline AGMO & Alkylglycerol Monooxygenase & $\begin{array}{l}\text { GO:0019432 (triglyceride } \\
\text { biosynthetic process) }\end{array}$ & Glucose & 0.094 & -16 & 0.021 & 0.33 & AOR \\
\hline APOC3 & Apolipoprotein C3 & $\begin{array}{l}\text { GO:0006641 (triglyceride } \\
\text { metabolic process) }\end{array}$ & Triglycerides & 0.0056 & -56 & 0.0065 & 0.38 & LIV \\
\hline DGAT2 & Diacylglycerol Acyltransferase 2 & $\begin{array}{l}\text { GO:0019432 (triglyceride } \\
\text { biosynthetic process) }\end{array}$ & Glucose & 0.049 & -19 & 0.024 & 0.56 & LIV \\
\hline DGAT2 & Diacylglycerol Acyltransferase 2 & $\begin{array}{l}\text { GO:0019432 (triglyceride } \\
\text { biosynthetic process) }\end{array}$ & Cholesterol & 0.078 & -24 & 0.036 & 0.089 & MAM \\
\hline GAA & Glucosidase Alpha, Acid & $\begin{array}{l}\text { GO:0006006 (glucose metabolic } \\
\text { process) }\end{array}$ & $\begin{array}{l}\mathrm{HDL} \\
\text { Cholesterol }\end{array}$ & 0.039 & 11 & 0.0021 & -0.21 & MAM \\
\hline PCSK9 & $\begin{array}{l}\text { Proprotein Convertase Subtilisin/ } \\
\text { Kexin Type } 9\end{array}$ & $\begin{array}{l}\text { GO:0008203 (cholesterol } \\
\text { metabolic process) }\end{array}$ & Glucose & 0.042 & -15 & 4.6E-05 & 0.65 & LIV \\
\hline PLCD4 & Phospholipase C Delta 4 & $\begin{array}{l}\text { GO:0006629 (lipid metabolic } \\
\text { process) }\end{array}$ & $\begin{array}{l}\text { Diastolic } \\
\text { Blood } \\
\text { Pressure }\end{array}$ & 0.030 & 1.9 & 0.027 & -2.6 & MAM \\
\hline
\end{tabular}

AOR aorta, MAM mammary artery, LIV liver, SF subcutaneous fat, SKLM skeletal muscle. $\beta$ values represent effect size. Positive $\beta$ in BioMe and negative $\beta$ in STARNET indicate LoF and low gene expression are associated with increased trait measurements. Negative $\beta$ in BioMe and positive $\beta$ in STARNET indicate LoF and low gene expression are associated with decreased trait measurements

(Fig. 4c). Corresponding positive and significant $(p<0.05)$ associations with plasma levels of glucose, cholesterol and triglycerides were also observed for hepatic DGAT2 expression in STARNET (Fig. 4d-f).

DGAT catalyzes the final step in triglyceride synthesis [43] and there are two known DGAT enzymes, DGAT1 and DGAT2. DGAT1 has been extensively investigated as a therapeutic target for obesity and obesity-related metabolic disorders such as insulin resistance. Across several preclinical studies [37, 44, 45], DGAT1 inhibitors were shown to reduce body weight and improve insulin sensitivity. In contrast, DGAT2 inhibition has not been extensively investigated. Among the limited studies performed so far, antisense oligonucleotides against DGAT2 lowered hepatic lipid levels in mice [46-48], however, pharmacological inhibition of DGAT2 with selective small molecule inhibitors has not been reported.

To evaluate the effect of specific inhibition of DGAT2 and to compare this with inhibition of DGAT1, we treated C57BL/6 mice with a highly selective DGAT1 small molecule inhibitor PF-04620110 (PF3) [49] or a highly selective DGAT2 inhibitor PF-06424439 (PF9) [38]. Treatment with the DGAT1 inhibitor was associated with significantly decreased body weight (Fig. 4g) and fasting glucose levels (Fig. 4h), consistent with previous reports $[37,44]$. Similar effects were observed in mice treated with the DGAT2 inhibitor (Fig. $4 \mathrm{~g}$ and h). However, whereas weight loss following DGAT2 inhibition was borderline significant (Fig. 4g), the lowering of fasting plasma glucose levels was more pronounced than in mice treated with the DGAT1 inhibitor (Fig. 4h).

\section{Discussion}

In drug discovery and development, half of all therapeutics fail in phase 2 clinical trials due to lack of efficacy [50]. This is immensely expensive and pharmaceutical companies are urgently looking for new strategies to better select targets at an earlier stage. In this regard, the power and accuracy of human genetics for target selection is increasingly being recognized [11]. In the current study, we identified and cataloged 2818 LoFs in 2143 genes from genotyping and the imputed data in the Mount Sinai BioMe Biobank [51]. LoFs in 433 of these genes were found to be associated with at least one of 10 major CVD traits in clinical data of the MHS-EMRs after careful annotation. 115 genes were further confirmed as being concordantly associated with the same trait in the world's largest human RNAseq datasets from blood, vascular, and metabolic tissues in STARNET [27]. Although the genetic data in $\mathrm{Bio} M e$ is based on genotyping 


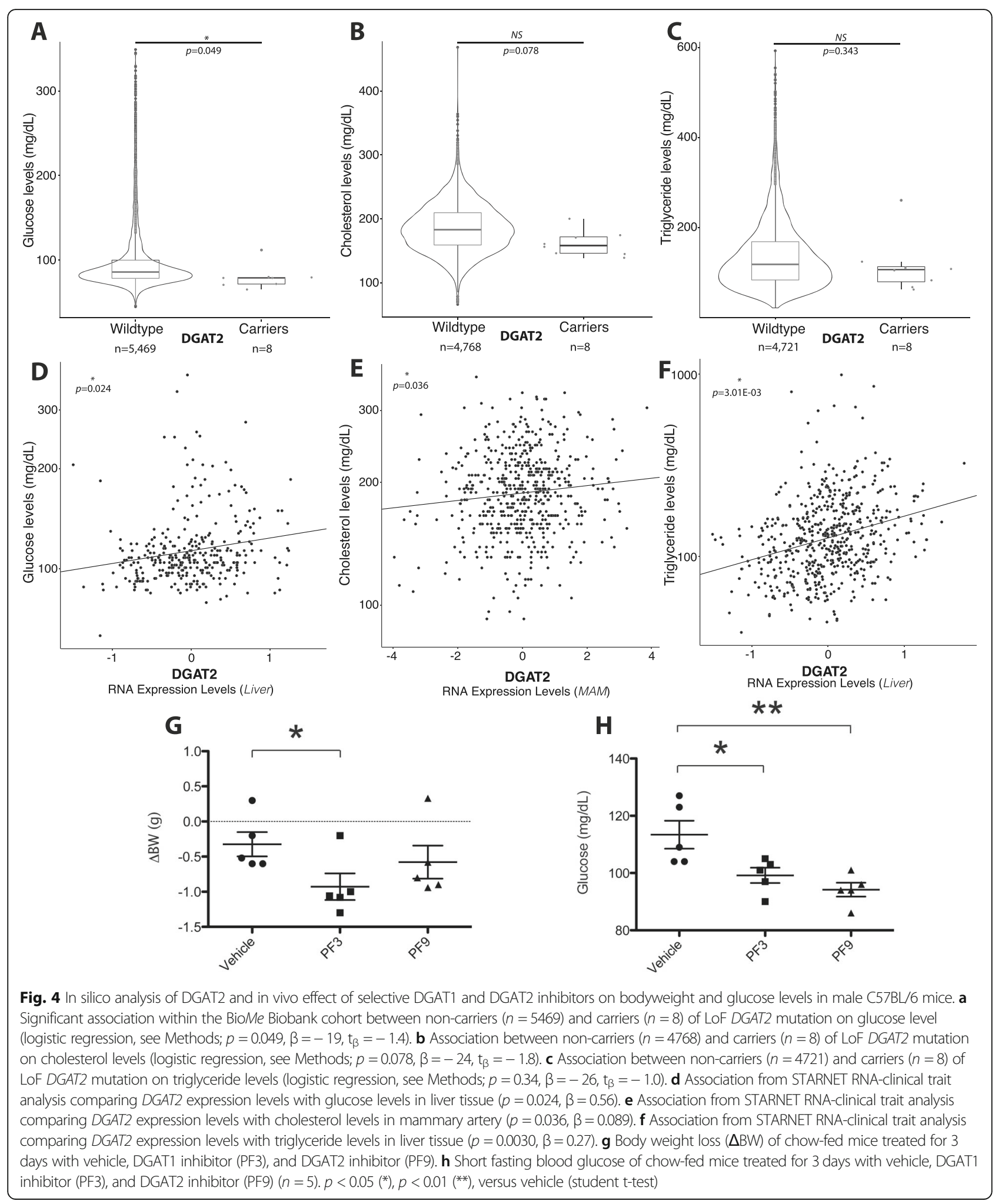

and imputation, the majority (75\%) of the analyzed 2818 LoFs and $82 \%$ of the LoFs in the 115 genes with concordant associations were genotyped, enhancing our confidence in the results. Using complementary data- and knowledge- driven approaches, we then successfully validated five novel liver genes for lowering of plasma cholesterol and triglycerides in HepG2 cells. In addition, we revealed that DGAT2 inhibitors have marked effects on plasma glucose in vivo. 
This represents the first systematic study of a large, hospital-based EMR-linked biobank to uncover and catalog novel LoFs associated with CVD traits that were then validated in global gene expression data. As such, it serves to improve our understanding of CVD biology and highlights novel putative CVD target genes that merit further investigation.

In comparison to the DiscovEHR study [19] where 176,000 LoFs were identified from 50,726 exomes, we identified significantly fewer LoFs in the BioMe cohort. Besides a smaller sample size and different makeup of ethnicity, the reason for the large discrepancy in predicted number of LoFs between the studies is that we used genotyping and the imputed data. Nearly $60 \%$ of the LoFs detected in the DiscovEHR cohort are singletons and $98.5 \%$ had an allele frequency of $<0.1 \%$. With respect to associations between LoFs and clinical traits, the DiscovEHR study only focused on nine therapeutic targets with drugs approved by the FDA or in clinical trials. In contrast, we validated novel gene-trait associations in independent gene expression data and carried out in vitro and in vivo functional studies.

We identified six novel candidate CVD-related genes using our pipeline. For these six genes we found strong support for their roles in hepatic control of plasma cholesterol (PCK2, RNMTL1 and SCRN2) and triglyceride (ABHD14B, NMRAL1 and SLC39A5) levels using gene silencing in HepG2 cells (Fig. 3). None of the three plasma cholesterol-lowering candidate genes have previously been implicated in hepatic cholesterol homeostasis (see Supplementary Discussion). Given the pronounced and concordant effects of silencing PCK2, RNMTL1, and SCRN2 in reducing media levels of APOB100 and PCSK9 and increasing the expression of LDLR in HepG2 cells (all but PCK2) these genes should be further evaluated as novel targets for lowering plasma cholesterol.

LoFs in three previously unassociated genes (ABDH14B, NMRAL1 and SLC39A5), the documented plasma triglyceride-related genes, $A P O C 3[52,53]$ and CES3 $[40,41]$ were found to be associated with lower plasma triglyceride levels in $\mathrm{Bio} M e$ and STARNET liver data. Again using gene silencing in HepG2 cells (Fig. 3), we not only found strong support for this role of $A P O C 3$ but also equally for SLC39A5, with concurrent and marked effects on levels of both media and intracellular triglycerides and media APOB100 (Fig. 3d). Silencing of $A B D H 14 B$ and NMRAL1 affected APOB100 secretion in opposite ways but had no effect on either media or cellular triglycerides. Taken together, our validation confirms the role of $A P O C 3$, and strongly suggests that SLC39A5 may be an interesting candidate gene for lowering plasma triglyceride levels. For NMRAL1 and $A B D H D 14 B$, marked and opposite effect were observed on media APOB100 whereas media and cellular triglyceride levels were unaffected. Thus the roles of these two genes require further scrutiny.

Our study also provided human genetic support for DGAT2 as a therapeutic target, which has not previously been reported. Although both DGAT1 and DGAT2 catalyze the same reaction in the last step of triglyceride synthesis, often in the same cells, DGAT1 but not DGAT2 has been pursued for more than a decade as a therapeutic target for obesity and metabolic disorders such as insulin resistance. This is primarily because DGAT1 mouse knockout (KO) models are resistant to diet-induced obesity, show decreased levels of tissue triglycerides, and have increased sensitivity to insulin and leptin [54, 55]. In contrast, DGAT2 KO mice die soon after birth, apparently from profound reductions in energy metabolism substrates and impaired skin permeability [56]. Although DGAT1 inhibitors consistently demonstrate metabolic benefit in various animal models [37, 44, 45], results from several clinical trials have been disappointing [57]. Specifically, while most of the clinical candidates exhibited the desired pharmacokinetic properties and target engagement, they failed to show effects on GLP-1 levels and insulin sensitivity. Moreover, there were gastrointestinal side effects such as nausea, diarrhea, and vomiting $[57,58]$. Indeed, it was later discovered that a rare LoF splice site mutation in DGAT1 in an Ashkenazi Jewish family is linked to a congenital diarrheal disorder (CDD) [59]. Therefore, the lack of success from inhibiting DGAT1 in humans, despite promising results from animal models, may be explained by the differing phenotypes of DGAT1 deficiency in human and mouse, again emphasizing the significance of target validation using human genetics. Association of LoF mutations or reduced expression in DGAT2 with reduced plasma cholesterol and glucose levels in both BioMe and STARNET cohorts in this study, coupled with the observation that a selective DGAT2 inhibitor had similar effects in mice as DGAT1 inhibitors (Fig. 4), suggests that DGAT2 could be a more preferable therapeutic target in humans than DGAT1.

We recognize there are several limitations to our study. First, as noted above, biobank data is not generated based upon on a prospective design for specific diseases or disease-relevant traits, hence clinical data quality is a concern. Even though we developed a thorough and individualized QC process for the 10 analyzed traits, with manual curation by two cardiologists to compile a list of medications and their related diseases that may affect each trait, there could be other factors affecting trait measurements that were not accounted for. These may include unreported medication use or 
pathophysiologic conditions when the measurements were taken. The sample size is another limitation with biobank data. While we analyzed associations of LoF genotypes with $10 \mathrm{CVD}$ and metabolic traits, we also attempted to perform similar association analysis on risk for CVDs. However, due to small sample sizes for each specific disease, the analysis did not yield interpretable results and is thus omitted from this report. Second, computational prediction of LoFs is still an empiric, imperfect process. Without experimental confirmation of each predicted LoF, there may be false positive and false negative associations for each gene. It is conceivable that some missense mutations lead to diminished or complete loss of protein function, but they are not included in our analysis. This is potentially problematic because LoFs are relatively rare in the human genome, and any false positive or false negative annotations may significantly impact the sample size of the case group (i.e. LoF carriers), causing identification of spurious LoF-trait associations. Third, genetic associations with diseases or disease related traits are often dependent upon racial and ethnic background. While the BioMe Biobank is racially diverse, the STARNET patients are predominantly White Caucasians. Thus, lack of validation in the STARNET cohort does not necessarily indicate the LoF/CVD-trait associations identified the BioMe Biobank cohort are false. However, genes with concordant CVD-trait associations in both BioMe Biobank and STARNET merit further scrutiny. In addition, due to the relatively low frequency of many of the variants characterized in this study coupled with the sample size of the BioMe Biobank cohort, we were underpowered to perform any form of stratified analysis to assess the contribution of racial or ethnic background to these associations. Lastly, we elected to use uncorrected, lenient $p$-value thresholds for both BioMe and STARNET data analyses $(p<0.1$ and $p<0.05$, respectively) with the understanding that we were less concerned with specific individual associations but more with those that were orthogonally significant in multiple datasets and validated experimentally.

\section{Conclusion}

In conclusion by leveraging the unique EMR-linked BioMe Biobank, we were able to identify and catalog 433 LoF-harboring genes found to be associated with at least one of 10 major CVD traits. Next and in contrast to recently published genotype-phenotype association studies $[15,19,60]$, we successfully validated 115 of these putative gene-CVD associations using STARNET RNA-seq data from seven metabolic and vascular tissues. As a result, experimental in vitro validations of PCK2, RNMTL1 and SCRN2, and
$A B H D 14 B, N M R A L 1$ and SLC39A confirmed roles for these genes in regulating plasma levels of cholesterol and triglycerides, respectively. Moreover, hepatic DGAT2 merits further attention as a parallel, or possibly alternative, drug target to DGAT1 for improving insulin resistance and reducing body weight.

\section{Additional files}

Additional file 1: Supplementary Material, Supplementary Methods, Supplementary Results, Supplementary Discussion, Supplementary Figure legends, Supplementary Table legends; Figures S1-S9. (PDF 8049 kb)

Additional file 2: Tables S1-S9. (XLSX $1175 \mathrm{~kb}$ )

\section{Abbreviations}

CVD: Cardiovascular disease; EMR: Electronic medical record; HDL: Highdensity lipoprotein; LDL: Low-density lipoprotein; LoF: Loss of function

\section{Acknowledgements}

This work was supported in part through the computational resources and staff expertise provided by the Department of Scientific Computing at the Icahn School of Medicine at Mount Sinai. The BioMe Biobank was funded by The Charles Bronfman Institute for Personalized Medicine at the Icahn School of Medicine at Mount Sinai. We thank Drs. Erick Scott and Ruth Loos for valuable discussions, and Dr. Jörg Hakenberg for technical assistance.

\section{About this supplement}

This article has been published as part of BMC Medical Genomics Volume 12 Supplement 6, 2019: Proceedings of Varl-COSI 2018: identification and annotation of genetic variants in the context of structure, function, and disease: medical genomics. The full contents of the supplement are available online at https://bmcmedgenomics.biomedcentral.com/articles/supplements/volume12-supplement-6.

\section{Authors' contributions}

RC conceived the study; RC, JLMB, SDL, CC, and JCK designed and supervised the study; BSG and NKA performed data analysis; LA performed in vitro experiments; HR performed in vivo experiments; OF, LL, GMB, KLA, KS, $M A B, K W J, B R, B J D, E E K, C B, K S, R E$, JS, AR, EES, JTD contributed to study design, data generation, data analysis and interpretation; BSG, LA, HR, JCK, $C C, S D L, J L M B$ and $R C$ wrote and revised the manuscript. All authors have read and approved the manuscript.

\section{Funding}

This work was funded by the Icahn School of Medicine at Mount Sinai. We acknowledge research support from the National Institutes of Health: [U54CA189201, UL1TR000067] to J.T.D., [R01HL130423] to J.C.K.,

[K23HL111339, R03HL135289] to C.G., [R21TR001739] to C.G. and J.L.M.B., [R01HL125863] to J.L.M.B.; the Transatlantic Network of Excellence Awards of The Leducq Foundation Consortium "CAD Genomics" to C.G. and J.L.M.B. and "Cellular and Molecular Targets to Promote Therapeutic Cardiac Regeneration" to J.C.K. Publication costs are funded by Sema4, a Mount Sinai venture, Stamford, CT 06902, USA.

Availability of data and materials

Access to data in BioMe and STARNET can be achieved through formal request using the database of Genotypes and Phenotypes (dbGaP) using study accessions phs000925.v1.p1 and phs001203.v1.p1 respectively.

Ethics approval and consent to participate

The study protocol was approved by the Mount Sinai Institutional Review Board (IRB), and conducted in accordance with the Declaration of Helsinki and Good Clinical Practice (GCP). Informed consent was obtained from all patients.

Consent for publication

Not applicable. 


\section{Competing interests}

$\mathrm{KLA}, \mathrm{EES}, \mathrm{SDL}$, and RC are employees of Sema4, a Mount Sinai venture. OF, $A R$, EES, and JLMB are employees of Clinical Gene Networks AB. The rest of authors declare they have no competing interest.

\section{Author details}

'Department of Genetics and Genomic Sciences, The Icahn Institute for Genomics and Multiscale Biology, Icahn School of Medicine at Mount Sinai, One Gustave L. Levy Place, New York, NY 10029, USA. ${ }^{2}$ The Institute for Next Generation Healthcare, Icahn School of Medicine at Mount Sinai, One Gustave L. Levy Place, New York, NY 10029, USA. ${ }^{3}$ Cardiovascular Research Center and Cardiovascular Institute, Icahn School of Medicine at Mount Sinai, One Gustave L. Levy Place, New York, NY 10029, USA. ${ }^{4}$ Department of Pathophysiology, Institute of Biomedicine and Translation Medicine, University of Tartu, Biomeedikum, Ravila 19, 50411 Tartu, Estonia. ${ }^{5}$ Clinical Gene Networks AB, Jungfrugatan 10, 11444 Stockholm, Sweden. ${ }^{6}$ Charles Bronfman Institute of Personalized Medicine, Icahn School of Medicine at Mount Sinai, One Gustave L. Levy Place, New York, NY 10029, USA. 'Sema4, a Mount Sinai venture, Stamford, CT 06902, USA. ${ }^{8}$ Department of Preventive Medicine, Icahn School of Medicine at Mount Sinai, One Gustave L. Levy Place, New York, NY 10029, USA. ${ }^{9}$ Department of Immunology, Genetics and Pathology, Uppsala University, 75185 Uppsala, Sweden. ${ }^{10}$ Department of Cardiac Surgery, Tartu University Hospital, 1a Ludwig Puusepa Street, 50406 Tartu, Estonia. ${ }^{11}$ Integrated Cardio Metabolic Centre, Department of Medicine, Karolinska Institutet, Karolinska Universitetssjukhuset Huddinge, 141 86 Stockholm, Sweden. ${ }^{12}$ Department of Health Policy and Research, Icahn School of Medicine at Mount Sinai, One Gustave L. Levy Place, New York, NY 10029, USA. ${ }^{13}$ Department of Pediatrics, Herman B Wells Center for Pediatric Research, Center for Diabetes and Metabolic Diseases, Stark Neurosciences Research Institute, Indiana University, 635 Barnhill Dr., MS2049, Indianapolis, IN 46202, USA. ${ }^{14}$ Bakar Computational Health Sciences Institute, University of California San Francisco, San Francisco 94158, CA, USA. ${ }^{15}$ Integrated Cardio Metabolic Centre, Department of Medicine, Karolinska Institutet, Novum, 14157 Huddinge, Sweden.

Published: 25 July 2019

\section{References}

1. Cohen JC, Boerwinkle E, Mosley TH Jr, Hobbs HH. Sequence variations in PCSK9, low LDL, and protection against coronary heart disease. N Engl J Med. 2006;354(12):1264-72. https://doi.org/10.1056/NEJMoa054013.

2. Everett BM, Smith RJ, Hiatt WR. Reducing LDL with PCSK9 inhibitors--the clinical benefit of lipid drugs. N Engl J Med. 2015;373(17):1588-91. https:// doi.org/10.1056/NEJMp1508120.

3. Robinson JG, Farnier M, Krempf M, Bergeron J, Luc G, Averna M, et al. Efficacy and safety of alirocumab in reducing lipids and cardiovascular events. N Engl J Med. 2015;372(16):1489-99. https://doi.org/10.1056/ NEJMoa1501031.

4. Sabatine MS, Giugliano RP, Wiviott SD, Raal FJ, Blom DJ, Robinson J, et al. Efficacy and safety of evolocumab in reducing lipids and cardiovascular events. N Engl J Med. 2015;372(16):1500-9. https://doi.org/10.1056/ NEJMoa1500858.

5. Sabatine MS, Giugliano RP, Keech AC, Honarpour N, Wiviott SD, Murphy SA, et al. Evolocumab and clinical outcomes in patients with cardiovascular disease. N Engl J Med. 2017;376(18):1713-22. https://doi.org/10.1056/ NEJMoa1615664.

6. Stitziel NO, Won HH, Morrison AC, Peloso GM, Do R, Lange LA, et al. Inactivating mutations in NPC1L1 and protection from coronary heart disease. N Engl J Med. 2014;371(22):2072-82. https://doi.org/10.1056/ NEJMoa1405386.

7. Cannon CP, Blazing MA, Giugliano RP, McCagg A, White JA, Theroux P, et al. Ezetimibe added to statin therapy after acute coronary syndromes. N Engl J Med. 2015;372(25):2387-97. https://doi.org/10.1056/ NEJMoa1410489.

8. Kathiresan S. Developing medicines that mimic the natural successes of the human genome: lessons from NPC1L1, HMGCR, PCSK9, APOC3, and CETP. J Am Coll Cardiol. 2015;65(15):1562-6. https://doi.org/10.1016/j. jacc.2015.02.049.

9. Soutar AK, Naoumova RP. Mechanisms of disease: genetic causes of familial hypercholesterolemia. Nature Clin Pract Cardiovasc Med. 2007:4(4):214-25. https://doi.org/10.1038/ncpcardio0836.
10. Baigent C, Keech A, Kearney PM, Blackwell L, Buck G, Pollicino C, et al. Efficacy and safety of cholesterol-lowering treatment: prospective meta-analysis of data from 90,056 participants in 14 randomised trials of statins. Lancet. 2005; 366(9493):1267-78. https:/doi.org/10.1016/s0140-6736(05)67394-1.

11. Nelson MR, Tipney H, Painter JL, Shen J, Nicoletti P, Shen Y, et al. The support of human genetic evidence for approved drug indications. Nat Genet. 2015;47(8):856-60. https://doi.org/10.1038/ng.3314.

12. MacArthur DG, Balasubramanian S, Frankish A, Huang N, Morris J, Walter K, et al. A systematic survey of loss-of-function variants in human proteincoding genes. Science (New York, NY). 2012;335(6070):823-8. https://doi. org/10.1126/science.1215040.

13. Sulem $P$, Helgason $H$, Oddson A, Stefansson H, Gudjonsson SA, Zink F, et al. Identification of a large set of rare complete human knockouts. Nat Genet. 2015;47(5):448-52. https://doi.org/10.1038/ng.3243.

14. Gudbjartsson DF, Helgason H, Gudjonsson SA, Zink F, Oddson A, Gylfason A et al. Large-scale whole-genome sequencing of the Icelandic population. Nat Genet. 2015;47(5):435-44. https://doi.org/10.1038/ng.3247.

15. Li AH, Morrison AC, Kovar C, Cupples LA, Brody JA, Polfus LM, et al. Analysis of loss-of-function variants and 20 risk factor phenotypes in 8,554 individuals identifies loci influencing chronic disease. Nat Genet. 2015;47(6): 640-2. https://doi.org/10.1038/ng.3270.

16. Lim ET, Wurtz P, Havulinna AS, Palta P, Tukiainen T, Rehnstrom K, et al. Distribution and medical impact of loss-of-function variants in the Finnish founder population. PLoS Genet. 2014;10(7):e1004494. https://doi.org/10. 1371/journal.pgen.1004494.

17. Narasimhan VM, Hunt KA, Mason D, Baker CL, Karczewski KJ, Barnes MR, et al. Health and population effects of rare gene knockouts in adult humans with related parents. Science (New York, NY). 2016;352(6284):474-7. https:// doi.org/10.1126/science.aac8624.

18. Walter K, Min JL, Huang J, Crooks L, Memari Y, McCarthy S, et al. The UK10K project identifies rare variants in health and disease. Nature. 2015;526(7571): 82-90. https://doi.org/10.1038/nature14962.

19. Dewey FE, Murray MF, Overton JD, Habegger L, Leader JB, Fetterolf SN, et al. Distribution and clinical impact of functional variants in 50,726 wholeexome sequences from the DiscovEHR study. Science (New York, NY). 2016; 354(6319). https://doi.org/10.1126/science.aaf6814.

20. Nadkarni GN, Galarneau G, Ellis SB, Nadukuru R, Zhang J, Scott SA, et al. Apolipoprotein L1 variants and blood pressure traits in African Americans. J Am Coll Cardiol. 2017;69(12):1564-74. https://doi.org/10. 1016/j.jacc.2017.01.040.

21. Belbin GM, Odgis J, Sorokin EP, Yee MC, Kohli S, Glicksberg BS, et al. Genetic identification of a common collagen disease in puerto ricans via identityby-descent mapping in a health system. Elife. 2017;6. https://doi.org/10. 7554/eLife.25060.

22. Li L, Cheng WY, Glicksberg BS, Gottesman O, Tamler R, Chen R, et al. Identification of type 2 diabetes subgroups through topological analysis of patient similarity. Sci Transl Med. 2015;7(311):311ra174. https://doi.org/10. 1126/scitranslmed.aaa9364.

23. Glicksberg BS, Li L, Cheng WY, Shameer K, Hakenberg J, Castellanos R, et al. An integrative pipeline for multi-modal discovery of disease relationships. In: Pacific symposium on Biocomputing Pacific symposium on Biocomputing; 2015. p. 407-18.

24. Glicksberg BS, Li L, Badgeley MA, Shameer K, Kosoy R, Beckmann ND, et al. Comparative analyses of population-scale phenomic data in electronic medical records reveal race-specific disease networks. Bioinformatics. 2016; 32(12):i101-i10. https://doi.org/10.1093/bioinformatics/btw282.

25. Badgeley MA, Shameer K, Glicksberg BS, Tomlinson MS, Levin MA, McCormick PJ, et al. EHDViz: clinical dashboard development using opensource technologies. BMJ Open. 2016;6(3):e010579. https://doi.org/10.1136/ bmjopen-2015-010579.

26. Shameer K, Glicksberg BS, Hodos R, Johnson KW, Badgeley MA, Readhead B, et al. Systematic analyses of drugs and disease indications in RepurposeDB reveal pharmacological, biological and epidemiological factors influencing drug repositioning. Brief Bioinform. 2017. https://doi.org/10.1093/bib/bbw136.

27. Franzen O, Ermel R, Cohain A, Akers NK, Di Narzo A, Talukdar HA, et al. Cardiometabolic risk loci share downstream cis- and trans-gene regulation across tissues and diseases. Science (New York, NY). 2016;353(6301):827-30. https://doi.org/10.1126/science.aad6970.

28. Delaneau O, Marchini J, Zagury JF. A linear complexity phasing method for thousands of genomes. Nat Methods. 2012;9(2):179-81. https://doi.org/10. 1038/nmeth.1785 
29. Howie B, Fuchsberger C, Stephens M, Marchini J, Abecasis GR. Fast and accurate genotype imputation in genome-wide association studies through pre-phasing. Nat Genet. 2012;44(8):955-9. https://doi.org/10. 1038/ng.2354

30. 1000 Genomes Project Consortium, Auton A, Brooks LD, Durbin RM, Garrison EP, Kang HM, et al. A global reference for human genetic variation. Nature. 2015;526(7571):68-74. https://doi.org/10.1038/ nature15393.

31. Purcell S, Neale B, Todd-Brown K, Thomas L, Ferreira MA, Bender D, et al. PLINK: a tool set for whole-genome association and population-based linkage analyses. Am J Hum Genet. 2007;81(3):559-75. https://doi.org/10 1086/519795.

32. Cingolani $\mathrm{P}$, Platts $\mathrm{A}$, Wang le $\mathrm{L}$, Coon $\mathrm{M}$, Nguyen $\mathrm{T}$, Wang $\mathrm{L}$, et al. A program for annotating and predicting the effects of single nucleotide polymorphisms, SnpEff: SNPs in the genome of Drosophila melanogaster strain w1118; iso-2; iso-3. Fly. 2012;6(2):80-92. https://doi. org/10.4161/fly.19695.

33. Dobin A, Davis CA, Schlesinger F, Drenkow J, Zaleski C, Jha S, et al. STAR: ultrafast universal RNA-seq aligner. Bioinformatics. 2013;29(1):15-21. https://doi.org/10.1093/bioinformatics/bts635.

34. Harrow J, Frankish A, Gonzalez JM, Tapanari E, Diekhans M, Kokocinski $F$, et al. GENCODE: the reference human genome annotation for the ENCODE project. Genome Res. 2012;22(9):1760-74. https://doi.org/10. 1101/gr.135350.111.

35. Anders S, Pyl PT, Huber W. HTSeq--a Python framework to work with highthroughput sequencing data. Bioinformatics. 2015;31(2):166-9. https://doi. org/10.1093/bioinformatics/btu638.

36. Law CW, Chen Y, Shi W, Smyth GK. Voom: precision weights unlock linear model analysis tools for RNA-seq read counts. Genome Biol. 2014;15(2):R29. https://doi.org/10.1186/gb-2014-15-2-r29.

37. Cao J, Zhou Y, Peng H, Huang X, Stahler S, Suri V, et al. Targeting acyl-CoA diacylglycerol acyltransferase 1 (DGAT1) with small molecule inhibitors for the treatment of metabolic diseases. J Biol Chem. 2011;286(48):41838-51. https://doi.org/10.1074/jbc.M111.245456.

38. Futatsugi K, Kung DW, Orr ST, Cabral S, Hepworth D, Aspnes G, et al. Discovery and optimization of Imidazopyridine-based inhibitors of diacylglycerol acyltransferase 2 (DGAT2). J Med Chem. 2015;58(18):7173-85. https://doi.org/10.1021/acs.jmedchem.5b01006.

39. Lek M, Karczewski KJ, Minikel EV, Samocha KE, Banks E, Fennell T, et al. Analysis of protein-coding genetic variation in 60,706 humans. Nature. 2016; 536(7616):285-91. https://doi.org/10.1038/nature19057.

40. Lian J, Wei E, Wang SP, Quiroga AD, Li L, Di Pardo A, et al. Liver specific inactivation of carboxylesterase 3/triacylglycerol hydrolase decreases blood lipids without causing severe steatosis in mice. Hepatology (Baltimore, Md). 2012;56(6):2154-62. https://doi.org/10.1002/hep.25881.

41. Wei E, Ben Ali Y, Lyon J, Wang H, Nelson R, Dolinsky W, et al. Loss of TGH/ Ces3 in mice decreases blood lipids, improves glucose tolerance, and increases energy expenditure. Cell Metab. 2010;11(3):183-93. https://doi.org/ 10.1016/j.cmet.2010.02.005.

42. Sundaram M, Zhong S, Bou Khalil M, Links PH, Zhao Y, lqbal J, et al. Expression of apolipoprotein C-III in McA-RH7777 cells enhances VLDL assembly and secretion under lipid-rich conditions. J Lipid Res. 2010;51(1): 150-61. https://doi.org/10.1194/m900346-jlr200.

43. Farese RV Jr, Cases S, Smith SJ. Triglyceride synthesis: insights from the cloning of diacylglycerol acyltransferase. Curr Opin Lipidol. 2000;11(3): 229-34.

44. Liu J, Gorski JN, Gold SJ, Chen D, Chen S, Forrest G, et al. Pharmacological inhibition of diacylglycerol acyltransferase 1 reduces body weight and modulates gut peptide release--potential insight into mechanism of action. Obesity (Silver Spring, Md). 2013;21(7):1406-15. https://doi.org/10.1002/oby.20193.

45. Maciejewski BS, LaPerle JL, Chen D, Ghosh A, Zavadoski WJ, McDonald TS, et al. Pharmacological inhibition to examine the role of DGAT1 in dietary lipid absorption in rodents and humans. Am J Physiol Gastrointest Liver Physiol. 2013;304(11):G958-69. https://doi.org/10.1152/ ajpgi.00384.2012.

46. Choi CS, Savage DB, Kulkarni A, Yu XX, Liu ZX, Morino K, et al. Suppression of diacylglycerol acyltransferase-2 (DGAT2), but not DGAT1, with antisense oligonucleotides reverses diet-induced hepatic steatosis and insulin resistance. J Biol Chem. 2007;282(31):22678-88. https://doi.org/10.1074/jbc M704213200.
47. Liu Y, Millar JS, Cromley DA, Graham M, Crooke R, Billheimer JT, et al. Knockdown of acyl-CoA:diacylglycerol acyltransferase 2 with antisense oligonucleotide reduces VLDL TG and ApoB secretion in mice. Biochim Biophys Acta. 2008;1781(3):97-104. https://doi.org/10.1016/j. bbalip.2008.01.001.

48. Yu XX, Murray SF, Pandey SK, Booten SL, Bao D, Song XZ, et al. Antisense oligonucleotide reduction of DGAT2 expression improves hepatic steatosis and hyperlipidemia in obese mice. Hepatology (Baltimore, Md). 2005;42(2): 362-71. https://doi.org/10.1002/hep.20783.

49. Dow RL, Li JC, Pence MP, Gibbs EM, LaPerle JL, Litchfield J, et al. Discovery of PF-04620110, a potent, selective, and orally bioavailable inhibitor of DGAT-1. ACS Med Chem Lett. 2011;2(5):407-12. https://doi.org/10.1021/ ml200051p

50. Khanna I. Drug discovery in pharmaceutical industry: productivity challenges and trends. Drug Discov Today. 2012;17(19-20):1088-102. https://doi.org/10. 1016/j.drudis.2012.05.007

51. Streicher SA, Sanderson SC, Jabs EW, Diefenbach M, Smirnoff M, Peter I, et al. Reasons for participating and genetic information needs among racially and ethnically diverse biobank participants: a focus group study. J Community Genet. 2011;2(3):153-63. https://doi.org/10.1007/s12687011-0052-2.

52. Jorgensen AB, Frikke-Schmidt R, Nordestgaard BG, Tybjaerg-Hansen A. Lossof-function mutations in APOC3 and risk of ischemic vascular disease. N Engl J Med. 2014;371(1):32-41. https://doi.org/10.1056/NEJMoa1308027.

53. Pollin TI, Damcott CM, Shen H, Ott SH, Shelton J, Horenstein RB, et al. A null mutation in human APOC3 confers a favorable plasma lipid profile and apparent cardioprotection. Science. 2008;322(5908):1702-5. https://doi.org/ 10.1126/science.1161524.

54. Chen HC, Smith SJ, Tow B, Elias PM, Farese RV Jr. Leptin modulates the effects of acyl CoA:diacylglycerol acyltransferase deficiency on murine fur and sebaceous glands. J Clin Invest. 2002;109(2):175-81. https://doi.org/10. 1172/jci13880.

55. Smith SJ, Cases S, Jensen DR, Chen HC, Sande E, Tow B, et al. Obesity resistance and multiple mechanisms of triglyceride synthesis in mice lacking Dgat. Nat Genet. 2000;25(1):87-90. https://doi.org/10.1038/75651.

56. Stone SJ, Myers HM, Watkins SM, Brown BE, Feingold KR, Elias PM, et al. Lipopenia and skin barrier abnormalities in DGAT2-deficient mice. J Biol Chem. 2004;279(12):11767-76. https://doi.org/10.1074/jbc.M311000200.

57. DeVita RJ, Pinto S. Current status of the research and development of diacylglycerol O-acyltransferase 1 (DGAT1) inhibitors. J Med Chem. 2013; 56(24):9820-5. https://doi.org/10.1021/jm4007033.

58. Denison H, Nilsson C, Lofgren L, Himmelmann A, Martensson G, Knutsson M, et al. Diacylglycerol acyltransferase 1 inhibition with AZD7687 alters lipid handling and hormone secretion in the gut with intolerable side effects: a randomized clinical trial. Diabetes Obes Metab. 2014;16(4):334-43. https:// doi.org/10.1111/dom.12221.

59. Haas JT, Winter HS, Lim E, Kirby A, Blumenstiel B, DeFelice M, et al. DGAT1 mutation is linked to a congenital diarrheal disorder. J Clin Invest. 2012; 122(12):4680-4. https://doi.org/10.1172/jci64873.

60. Abul-Husn NS, Manickam K, Jones LK, Wright EA, Hartzel DN, GonzagaJauregui C, et al. Genetic identification of familial hypercholesterolemia within a single U.S. health care system. Science (New York, NY). 2016; 354(6319). https://doi.org/10.1126/science.aaf7000.

\section{Publisher's Note}

Springer Nature remains neutral with regard to jurisdictional claims in published maps and institutional affiliations.

Ready to submit your research? Choose BMC and benefit from:

- fast, convenient online submission

- thorough peer review by experienced researchers in your field

- rapid publication on acceptance

- support for research data, including large and complex data types

- gold Open Access which fosters wider collaboration and increased citations

- maximum visibility for your research: over $100 \mathrm{M}$ website views per year

At $\mathrm{BMC}$, research is always in progress.

Learn more biomedcentral.com/submission 